\title{
Perspective on completing natural inflation
}

\author{
Ki-Young Choi ${ }^{1}$, Jihn E. Kim ${ }^{2,3}$ and Bumseok Kyae ${ }^{4 *}$ \\ ${ }^{1}$ Korea Astronomy and Space Science Institute, Daejeon, South Korea \\ ${ }^{2}$ Department of Physics, Seoul National University, Seoul, South Korea \\ ${ }^{3}$ Department of Physics, Kyung Hee University, Seoul, South Korea \\ ${ }^{4}$ Department of Physics, Pusan National University, Busan, South Korea
}

Edited by:

Nicolao Fornengo, University of Torino, Italy

Reviewed by:

Mar Bastero-Gil, Universidad de

Granada, Spain

Bhupal Dev, University of

Manchester, UK

*Correspondence:

Bumseok Kyae, Department of

Physics, Pusan National University,

Busan 609-735, South Korea

e-mail: bkyae@pusan.ac.kr
We present a perspective on the inflation paths in $2-, 3-, \ldots, N$-flation models based on the ultraviolet completion in heterotic string theory, where a number of grand unification scale axions are used. The number of non-Abelian gauge groups for a natural inflation is restricted in string compactification, and we argue that the most plausible completion of natural inflation from a theory perspective is the $2-$ and 3 -flation.

Keywords: natural inflation, 2-flation, N-flation, high scale inflation, GUT scale groups

\section{INTRODUCTION}

After the recent report by the BICEP2 group on a large tensorto-scalar ratio $r$ [1], natural inflation [2] and the method of completing it [3] have attracted a great deal of attention [4-9, 11] . Completing natural inflation employs at least two grand unification(GUT) scale non-Abelian gauge groups, which can be called $2-, 3-, \ldots, N-$ flation models. In this paper, we present a perspective on completing natural inflation.

Cosmic inflation is a paradigm for a solution of the homogeneity and flatness problems [12-14]. For a sufficient inflation with the e-fold number $e>(50-60)$, one needs small inflationparameters, $\epsilon\left(\equiv \frac{1}{2} M_{\mathrm{P}}^{2}\left(V^{\prime} / V\right)^{2}\right)$ and $\eta\left(\equiv M_{\mathrm{P}}^{2} V^{\prime \prime} / V\right)[15,16]$. Single bubble inflation were proposed with the initial condition near the origin in the Coleman-Weinberg type logarithmicallyflat hilltop potential [17], or at a large field value for a chaotic type potential [18]. With the slow-roll conditions satisfied, the local non-Gaussianities $\left|f_{\mathrm{NL}}^{\text {local }}\right|$ are much smaller than 1 for a single field inflation [19], which was reported by the Planck 2013 group [20]. In addition, if the BICEP2 result with a large $r$ is confirmed, the hybrid inflation predicting $n_{s}>1$ (arising from the hilltop inflation) [21] and the $\lambda \phi^{4}$ chaotic inflation are disfavored from the data [20]. Even though the size of dust contribution is still an ongoing issue [22], here we assume a large $r$ of order 0.1 .

The negligible non-Gaussianity pins down the inflation models to the single field $m^{2} \phi^{2}$ chaotic inflation $[4-9,11]$ or the multi-field hilltop inflation [21]. The $m^{2} \phi^{2}$ chaotic inflation needs a fine-tuning of order $m^{2} \approx 10^{-10}$ in units of the reduced Planck mass, $M_{\mathrm{P}} \simeq 2.44 \times 10^{18} \mathrm{GeV}$. For the predictability of the Einstein equation, we need that the potential $V$ during inflation must be much smaller than $M_{\mathrm{P}}^{4}$. In fact, this can be easily realized in natural inflation where there exists a GUT scale heavy axion coupling to a GUT scale confining force [2]. With the heavy axion potential at the GUT scale $\left(\approx \Lambda_{\mathrm{GUT}} \approx M_{\mathrm{GUT}}\right)$, the explicit breaking potential of the Peccei-Quinn (PQ) symmetry is given by $\propto \frac{1}{2} \Lambda_{\mathrm{GUT}}^{4}(1-\cos (a / f))$, where $a$ and $f$ denote the axion and its decay constant, respectively; thus the potential energy is bounded by $\Lambda_{\mathrm{GUT}}^{4}$.

The $m^{2} \phi^{2}$ chaotic inflation has a problem, "Why does one keep only the quadratic term?" It is known that a large transPlanckian field value is needed in the $m^{2} \phi^{2}$ chaotic inflation for a large tensor-to-scalar ratio $r$, which is known as the Lyth bound $\langle\phi\rangle>15 M_{\mathrm{P}}$ [23]. In particular, with the large trans-Planckian field value higher order terms might be more important [21]. To reconcile the trans-Planckian field value with the natural inflation idea, Kim, Nilles, and Peloso (KNP) introduced two axions and two confining forces at the GUT scale. It has been generalized to N-flation [24]. An ultra-violet completed theory, in particular the heterotic string theory, may not allow a large number of non-Abelian gauge groups. We scrutinize the inflaton path, arising from the limited rank of the total gauge group, and present an argument that 2-flation (possibly 3-flation also), i.e., the KNP type, is an easily realizable one.

In Section 2, we briefly review the KNP scenario and its Nflation extension. In Section 3, we discuss the maximum rank of the heterotic string, which is argued for a limitation of the number of GUT scale confining gauge groups. Section 4 is a conclusion.

\section{THE 2-FLATION}

A large vacuum expectation value (VEV) of a scalar field is possible with a small mass parameter if a very small coupling constant $\lambda$ is assumed,

$$
V=\frac{1}{4} \lambda\left(|\phi|^{2}-f^{2}\right)^{2}
$$

The mass parameter in this theory is $m^{2}=\lambda f^{2}$. With a GUT scale $m, f$ can be trans-Planckian of order $>10 M_{\mathrm{P}}$ for $\lambda<10^{-6}$. However, the potential (1) with the small $\lambda$ describes inflation 
starting from near the convex hilltop point (due to the high temperature effect before inflation) and hence it is not favored by the BICEP2 data [21]. This has led to the recent surge of studies on concave potentials near the origin of the field space in case of single field inflations $[4-9,11]$. The concave potentials give positive $\eta$ 's.

The simplest concave potential is the $m^{2} \phi^{2}$ chaotic potential. Since this potential is not bounded from above, the natural inflation with a GUT scale confining force has been introduced so that the potential is bounded from above [2] where the GUT scale axion is the inflaton and the inflaton potential is

$$
V=\Lambda_{G U T}^{4}\left(1-\cos \frac{a_{N}}{f_{N}}\right)
$$

With $\mathrm{O}(1)$ parameters at the GUT scale, $f_{N}$ is $\mathrm{O}\left(M_{\mathrm{GUT}}\right)$. This potential arises from $V=-\frac{1}{2}\left(\Lambda_{G U T}^{4}+A\right) e^{i a / f}+$ h.c. where $A$ is a function of a real scalar field $\rho$. So, the potential (2) mainly depends on the axion field $a$, and $f_{N}$ is not determined by Equation (2) but by the potential breaking the PQ symmetry spontaneously, i.e., by Equation (1). For the radial direction to roll down quickly, we need $\lambda \gg 10^{-6}$ and $f_{N}$ is of order $M_{\mathrm{GUT}}$.

Since we need a trans-Planckian value for the decay constant of the GUT axion, the KNP model has been proposed with two axions $a_{1}$ and $a_{2}$ and two GUT scale $\left(\Lambda_{1}\right.$ and $\left.\Lambda_{2}\right)$ confining forces, resulting in the following minus-cosine potential

$$
\begin{aligned}
V= & \Lambda_{1}^{4}\left(1-\cos \left[\alpha \frac{a_{1}}{f_{1}}+\beta \frac{a_{2}}{f_{2}}\right]\right) \\
& +\Lambda_{2}^{4}\left(1-\cos \left[\gamma \frac{a_{1}}{f_{1}}+\delta \frac{a_{2}}{f_{2}}\right]\right),
\end{aligned}
$$

where $\alpha, \beta, \gamma$, and $\delta$ are determined by two $\mathrm{U}(1)$ quantum numbers. Of course, $f_{1}$ and $f_{2}$ are $\mathrm{O}\left(M_{\mathrm{GUT}}\right)$. Let us comment on a few issues related to the above potential.

If there is only one confining force at the GUT scale, we can set $\Lambda_{2}=0$ in Equation (3). In this case, there exists a flat Goldstone boson direction as shown with the red valley in Figure 1A. The blue bullet field point of Figure 1A quickly rolls along the blue path down to the red line vacuum. This flat red line direction cannot work as an inflation direction, but it corresponds to an axion potential with an infinite axion decay constant. Thus, with two axions and two nonvanishing axion potentials of Equation (3), there is a possibility of obtaining a trans-Planckian decay constant.

If there are two confining forces with nonvanishing $\Lambda_{1}^{4}$ and $\Lambda_{2}^{4}$, the situation is shown in Figure 1B [3], with no Goldstone boson direction. The red line direction of Figure $\mathbf{1 A}$ is lifted. To mimic the large $f$ limit of Figure 1A, we choose a direction such that the PQ quantum numbers are aligned, and some people use the terminology 'aligned inflation' instead of natural inflation. The condition for the alignment is $\alpha \delta \approx \beta \gamma$. This is because two axion masses, the heavy axion $a_{h}$ and the light axion $a_{I}$, are

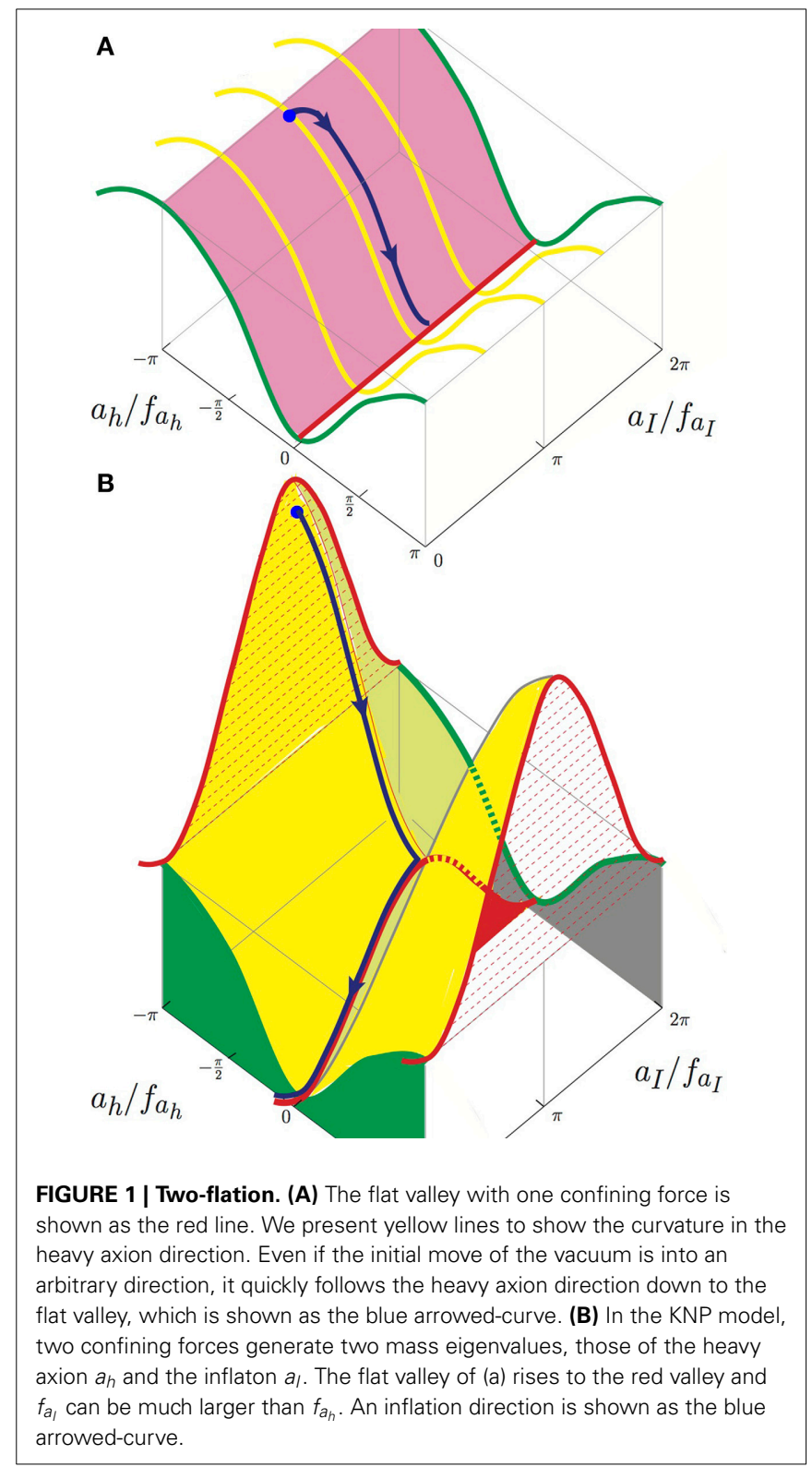

$$
\begin{aligned}
& m_{a_{h}}^{2}=\frac{1}{2}(A+B), \\
& m_{a_{I}}^{2}=\frac{1}{2}(A-B),
\end{aligned}
$$

where

$$
\begin{aligned}
& A=\frac{\alpha^{2} \Lambda_{1}^{4}+\gamma^{2} \Lambda_{2}^{4}}{f_{1}^{2}}+\frac{\beta^{2} \Lambda_{1}^{4}+\delta^{2} \Lambda_{2}^{4}}{f_{2}^{2}}, \\
& B=\sqrt{A^{2}-4(\alpha \delta-\beta \gamma)^{2} \frac{\Lambda_{1}^{4} \Lambda_{2}^{4}}{f_{1}^{2} f_{2}^{2}}} .
\end{aligned}
$$

Let the approximation, $\alpha \delta \approx \beta \gamma$, is described by a small number $\Delta$, i.e., $\alpha \delta=\beta \gamma+\Delta$. Then, the heavy axion and inflaton masses are 


$$
\begin{aligned}
& m_{a_{h}}^{2} \simeq \frac{\alpha^{2} \Lambda_{1}^{4}+\gamma^{2} \Lambda_{2}^{4}}{f_{1}^{2}}+\frac{\beta^{2} \Lambda_{1}^{4}+\delta^{2} \Lambda_{2}^{4}}{f_{2}^{2}}, \\
& m_{a_{I}}^{2} \simeq \frac{\Delta^{2} \Lambda_{1}^{4} \Lambda_{2}^{4}}{D}
\end{aligned}
$$

where $D=f_{2}^{2}\left(\alpha^{2} \Lambda_{1}^{4}+\gamma^{2} \Lambda_{2}^{4}\right)+f_{1}^{2}\left(\beta^{2} \Lambda_{1}^{4}+\delta^{2} \Lambda_{2}^{4}\right)$. For simplicity of discussion, we will set $\Lambda_{1}=\Lambda_{2}=\Lambda$ and $f_{1}=f_{2} \equiv f$. Then, the heavy and light masses are

$$
\begin{aligned}
& m_{a_{h}}^{2} \simeq\left(\alpha^{2}+\beta^{2}+\gamma^{2}+\delta^{2}\right) \frac{\Lambda^{4}}{f^{2}}, \\
& m_{a_{I}}^{2} \simeq \frac{\Lambda^{4}}{\left(\alpha^{2}+\beta^{2}+\gamma^{2}+\delta^{2}\right) f^{2} / \Delta^{2}},
\end{aligned}
$$

from which we obtain

$$
f_{a_{I}}=\frac{\sqrt{\alpha^{2}+\beta^{2}+\gamma^{2}+\delta^{2}} f}{|\Delta|} .
$$

With the same order of $\alpha, \beta, \gamma$, and $\delta$, the small number $\Delta$ can be $\mathrm{O}(1)$ to realize $f_{a_{I}} \approx 100 f$ if $\alpha, \beta, \gamma, \delta=\mathrm{O}(50)$. Thus, the probability for $\Delta \approx 1$ to be realized is 1 out of $50 \times 50$, i.e., the large $f_{a_{I}} \approx 100 f_{a_{h}}$ is possible in $0.04 \%$ of random PQ quantum numbers $\alpha, \beta, \gamma$, and $\delta$ of $\mathrm{O}(50)$. However, note that $\alpha, \beta, \gamma$, and $\delta$ are integer PQ quantum numbers. So, realizing the needed $2-$ flation is assigning $\alpha, \beta, \gamma$, and $\delta$ such that they are $\mathrm{O}(50)$. They are not random numbers but quantum numbers of singlet scalar fields. The question is how one can assign these large PQ quantum numbers. It belongs to a question on discrete symmetries [25]. If it is difficult to obtain such discrete symmetries, then one can say that the probability is small. If we do $\mathrm{N}$-flation with a large $\mathrm{N}$, then the PQ quantum numbers of $\mathrm{O}(2-10)$ are needed as briefly commented below, which may be easier to construct with discrete symmetries. However, the main perspective here is not on the PQ quantum numbers but are on the feasibility of obtaining a large numbers of non-Abelian gauge groups.

Suppose that we have a $\mathbf{Z}_{120}$ discrete symmetry. Then, singlet scalar fields can have quantum numbers of $0,1, \ldots, 119$. If this discrete symmetry is embedded in the $\mathrm{U}(1) \mathrm{PQ}$ symmetry, the PQ domain wall number $\alpha, \beta, \gamma$, and $\delta$ can be $0,1, \ldots, 119$. If the singlets having the PQ quantum numbers of $0,1, \ldots, 49$ and $71, \ldots, 119$ are not allowed to obtain VEVs, then $\alpha, \beta, \gamma$, and $\delta$ can be $50, \ldots, 70$. In this way, we obtain a desirable quantum numbers such that $\alpha \delta-\beta \gamma=O(1)$. This is not an issue of fine tuning but an issue on the VEVs of scalar fields.

We commented above that the flat valley of Figure $1 \mathrm{~A}$ rises to the red valley of Figure $1 \mathbf{B}$ and $f_{a_{I}}$ can be $\approx 100 f_{a_{h}}$ in a small region of the $\mathrm{PQ}$ quantum number space and the inflaton is the blue curve on top of the red valley.

\section{NUMBER AND SIZES OF NON-ABELIAN GAUGE GROUPS}

The KNP 2-flation model has been generalized to $\mathrm{N}$-flation models [24]. The N-flation has adopted two merits of 2-flation, one that the decay constant is $\approx \sqrt{2}$ times larger and the other that the maximum height of the potential is $\approx 2$ times larger, as depicted in Figure 1B. Namely, in the N-flation we expect that the decay constant can be $\approx \sqrt{N}$ times larger and the maximum height of the potential is $\approx N$ times larger. Then, from the highest point of the potential the denominators in the $\epsilon$ and $\eta$ calculation become $N$ times larger, making $\epsilon$ and $\eta N$ times smaller, and the decay constant is about $\sqrt{N}$ times larger. These merits are gradually diminished as the heavy axion paths shift directions as they roll down the hill. ${ }^{1}$.

In addition, in the $\mathrm{N}$-flation the PQ quantum numbers are not tuned to large values of $\mathrm{O}(50)$. However, an $\mathrm{N}$-flation with a large $N$ suffers from the theoretical requirement of introducing $N(\gg 2)$ GUT scale non-Abelian gauge groups. In obtaining $N$, we must satisfy the SM phenomenology also. After realizing the weak mixing angle, $\theta_{W}$ should be $\sin ^{2} \theta_{W}=\frac{3}{8}$ at the GUT scale [27], non-prime orbifold compactification became popular since $2004[28,29]$, and $\sin ^{2} \theta_{W}=\frac{3}{8}$ is possible in many non-prime orbifold GUTs. In general, $\sin ^{2} \theta_{W}=\frac{3}{8}$ was not easy to be realized in earlier orbifold models [30-33]. Successful SM construction have been obtained in the $\mathbf{Z}_{12-I}[34-36]$ and $\mathbf{Z}_{6-I I}$ orbifold compactifications [37-39]. However, heterotic string models have not provided a useful moduli stabilization program, even though there exist some suggestions on stabilization of some moduli [40$42]^{2}$. Dynamical supersymmetry breaking [45] would also be another issue in a 2-flation model with a rank 16 gauge group. In the heterotic string theory with level 1 construction, the sum of the ranks of gauge groups is 16 [or 22 in the Narain compactification $\left.[46]^{3}\right]$. Out of rank 16 , the SM uses 4 and rank 12 is left for the GUT scale confining gauge groups. If we use SU(4)'s for the $\mathrm{N}$-flation, the maximal $N$ is 4 . This is not a case that the $\mathrm{N}$ flation, motivated to have a large $N$, is aiming at. For $N=3$, the sum of the ranks of the GUT gauge groups is barely acceptable, taking into account another non-Abelian group for breaking supersymmetry. As commented before, cases of $N \geq 3$ reduce the PQ quantum number condition of the $N=2$ case. For example, to obtain $f_{a_{I}} \approx 100 f$, we need an approximate $10 \times 10 \times 10 \mathrm{PQ}$ quantum numbers (as we obtained Equation (11) for the $N=2$ case). Thus, the probability to obtain $f_{a_{I}} \approx 100 f$ is about $0.1 \%$. But note that this is the PQ quantum numbers which are not really random priors. It is the problem of vacuum expectation values of singlet scalars with an appropriate discrete symmetry.

In the D-brane construction of string theory, RamondRamond (RR) charges of D-branes should be canceled with proper orientifold p-planes ( $O_{p}$-planes), which can be regarded as the fixed planes under a $\mathbf{Z}_{2}$ symmetry: anti-D branes can also compensate the RR charge of D-branes, but they hardly break SUSY, making the system unstable. The RR charge of an $O_{p}$-plane $(p=0,1,2, \ldots, 9), Q_{O p}$ is given by $Q_{O p}=-2 \cdot 2^{p-5} \times Q_{D p}$, where $Q_{D p}$ denotes the RR charge of a $D p$ brane $e^{4}$. The maximum

\footnotetext{
${ }^{1}$ See, for example McDonald [26].

${ }^{2}$ For moduli stabilization in other frameworks, see e.g., Kachru et al. [43], Goldberger et al. [44].

${ }^{3}$ For level greater than 1 , it is possible to go beyond rank 16 . We are aware of one example of construction at level 3 [47], containing the SM gauge group (with suitable Higgs fields for breaking the gauge group) with three families of quarks and leptons. In principle, other string theories dual to the heterotic one with higher levels would also allow gauge groups whose rank is larger than 16. For instance, see [48].

${ }^{4}$ See, for example, Equation (15) of Giveon and Kutasov[49].
} 
$Q_{O p}$ is $\left.-32 Q_{D p}.\right]$ It can be canceled by a stack of $N_{c} D p$ branes (the maximum number is $32 Q_{D p}$ ) parallel to an $O p$-plane, which yield a rank $N_{c} / 2$ (the maximum number is 16) gauge group. Therefore, even in the D-brane construction, it is quite hard to obtain a gauge group whose rank is larger than 16 .

\section{CONCLUSION}

The idea of natural inflation, using a GUT scale axion, has been extended to include $2,3, \ldots, N$ axions. For the 2-flation, the PQ quantum numbers are almost degenerate, e.g. differing 1 out of 50. This almost degeneracy of the PQ quantum numbers can be relaxed by increasing $N$. In addition, the slow-roll parameters $\epsilon$ and $\eta$ can be reduced by a factor $1 / N$. Models along this line can be constructed at field theory level.

However, in string compactification the number of nonAbelian gauge groups are restricted, which makes the realization of N-flation very difficult. Most SM constructions from string compactification used the level 1 construction in which case the rank is 16. Even if higher levels are assumed, the rank is 22. In any case, the rank cannot be of order 100 . Because of this difficulty of obtaining a large number of non-Abelian GUT scale gauge groups, the easiest realization of the trans-Planckian decay constant is the 2- and 3-flation. Nevertheless, it will be interesting to find out $N \geq 4$ non-Abelian GUT scale gauge groups from string compactification with the features satisfying the low energy SM phenomenology.

\section{ACKNOWLEDGMENTS}

Ki-Young Choi is supported by the National Research Foundation (NRF) grant funded by the Korean Government (MEST) (No. 2011-0011083), Jihn E. Kim is supported in part by the NRF grant (No. 2005-0093841) and by the IBS(IBS CA1310), and Bumseok Kyae is supported in part by the NRF grant (No. 2013R1A1A2006904).

\section{REFERENCES}

1. Ade PAR, Aikin RW, Barkats D, Benton SJ, Bischoff CA, Bock JJ et al. BICEP2 I: Detection Of B-mode Polarization at Degree Angular Scales. Phys Rev Lett. (2014) 112: 241101. doi: 10.1103/PhysRevLett.112.241101

2. Freese K, Frieman JA, Orlinto AV. Natural inflation with pseudo Nambu-Goldstone bosons. Phys RevLett. (1990) 65: 3233-36. doi: 10.1103/PhysRevLett.65.3233

3. Kim JE, Nilles HP, Peloso M. Completing Natural Inflation. JCAP (2005) 0501: 005. doi: 10.1088/1475-7516/2005/01/005

4. Freese K, Kinney WH. Natural inflation: consistency with cosmic microwave background observations of planck and BICEP2. arXiv:1403.5277 [astroph.CO]

5. Kappl R, Krippendorf S, Nilles HP. Aligned Natural Inflation: Monodromies of two Axions. Phys Lett B (2014) 737: 124-8. doi: 10.1016/j.physletb.2014.08.045

6. Choi K, Kim H, Yun S. Natural inflation with multiple sub-Planckian axions. Phys RevD (2014) 90: 023545. doi: 10.1103/PhysRevD.90.023545

7. Dayan IB, Pedro FG, Westphal A, Wrase T. Towards natural inflation in string theory. arXiv:1407.2562 [hep-th]

8. Harigaya K, Ibe M, Yanagida TT. R-symmetric Axion/Natural Inflation in supergravity via deformed moduli dynamics. arXiv: 1409.0330[hep-ph]

9. Das K, Dutta K. N-flation in Supergravity. arXiv: 1408.6376[hep-ph]

10. Kim JE, Semertzidis YK, Tsujikawa S. Bosonic coherent motions in the Universe. Front Phys. (2014) 2: 60. doi: 10.3389/fphys.2014.00060

11. Li T, Li Z, Nanopoulos DV. Helical Phase Inflation. arXiv: 1409.3267[hep-ph]

12. Guth A. The inflationary universe: a possible solution to the Horizon and flatness problems. Phys Rev D (1981) 23: 347-56. doi: 10.1103/PhysRevD.23.347
13. Linde AD. A new inflationary universe scenario: a possible solution of the Horizon, flatness, homogeneity, isotropy and primordial monopole problems. Phys Lett B (1982) 108: 389-93. doi: 10.1016/0370-2693(82)91219-9

14. Albrecht A, Steinhardt PJ. Cosmology for grand unified theories with radiatively induced symmetry breaking. Phys RevLett. (1982) 48: 1220-3. doi: 10.1103/PhysRevLett.48.1220

15. Mukhanov V. Physical Foundations of Cosmology, Cambridge: Cambridge University Press.

16. Lyth DH, Riotto A. Particle physics models of inflation and the cosmological density perturbation. Phys Rep. (1999) 314: 1-146. doi: 10.1016/S03701573(98)00128-8

17. Coleman SR, Weinberg EJ. Radiative corrections as the origin of spontaneous symmetry breaking. Phys RevD (1973) 7: 1888-910. doi: 10.1103/PhysRevD.7.1888

18. Linde AD. Chaotic inflation. Phys LettB (1983) 129: 177-81. doi: 10.1016/0370-2693(83)90837-7

19. Tsujikawa S, Ohashi J, Kuroyanagi S, De Felice A. Planck constraints on single-field inflation. Phys RevD (2013) 88: 023529. doi: 10.1103/PhysRevD.88.023529

20. Ade PAR, Aghanim N, Armitage-Caplan C, Arnaud M, Ashdown M, Atrio-Barandela F et al. [Planck Collaboration]. Planck 2013 results. XXII. Constraints on inflation. [arXiv:1303.5082 [astro-ph.CO]].

21. Kim JE. The inflation point in $\mathrm{U}(1)_{\text {de }}$ hilltop potential assisted by chaoton, BICEP2 data, and trans-Planckian decay constant. Phys Lett B (2014) 737: 1-5. doi: 10.1016/j.physletb.2014.08.025

22. Ade PAR, Aghanim N, Arnaud M, Aumond J, Baccigalupi C, Banday AJ et al. [Planck Collaboration]. Planck intermediate results. XXX. The angular power spectrum of polarized dust emission at intermediate and high Galactic latitudes. arXiv: 1409.5738 [astro-ph.CO].

23. Lyth $\mathrm{DH}$. What would we learn by detecting a gravitational wave signal in the cosmic microwave background anisotropy?. Phys Rev Lett. (1997) 78: 1861-3. doi: 10.1103/PhysRevLett.78.1861

24. Dimopoulos S, Kachru S, McGreevy J, Wacker JG. N-flation. JCAP (2008) 0808: 003. doi: 10.1088/1475-7516/2008/08/003

25. Kim JE. Abelian discrete symmentries $\mathbf{Z}_{N}$ and $\mathbf{Z}_{n R}$ from string orbifolds. Phys Lett B (2013) 726: 450-5. doi: 10.1016/j.physletb.2013.08.039

26. McDonald J. A minimal sub-planckian axion inflation model with large tensor-to-scalar ratio. arXiv:1407.7471 [hep-ph]

27. Kim JE. $Z_{3}$ orbifold construction of SU $(3)^{3}$ GUT with $\sin ^{2} \theta_{W}=\frac{3}{8}$. Phys Lett $B$ (2003) 564: 35-41. doi: 10.1016/S0370-2693(03)00567-7

28. Choi K-S, Kim JE. Quarks and Leptons from Orbifolded Superstring. Lect Notes Phys. 696: 1-406. doi: 10.1007/b11681670

29. Kobayashi T, Raby S, Zhang R. Searching for realistic 4d string models with a Pati-Salam symmetry: Orbifold grand unified theories from heterotic string compactification on a $Z_{6}$ orbifold. Nucl Phys B (2004) 704: 3-55. doi: 10.1016/j.nuclphysb.2004.10.035

30. Ibañez LE, Kim JE, Nilles H P, Quevedo F. Orbifold Compactifications with Three Families of $\mathrm{SU}(3) \times \mathrm{SU}(2) \times \mathrm{U}(1)^{n}$. Phys Lett B (1987) 191: 282-6 doi: 10.1016/0370-2693(87)90255-3

31. Casas A, Munoz C. Three Generation $\mathrm{SU}(3) \times \mathrm{SU}(2) \times \mathrm{U}(1)_{\mathrm{Y}}$ Models from Orbifolds. Phys Lett B (1988) 209: 214-20 doi: 10.1016/0370-2693(88)90452-2

32. Ibañez LE. Computing the weak mixing angle from anomaly cancellation. Phys Lett B (1993) 303: 55-62. doi: 10.1016/0370-2693(93)90043-H

33. Ibañez LE. The Weak mixing angle in string theory and the Green-Schwarz mechanism. In: 23rd Eloisatron Workshop on Properties of SUSY particles, Erice, 1992: 54-64. arXiv: hep-ph/9303244

34. Kim JE, Kyae B. Flipped $\mathrm{SU}(5)$ from $\mathrm{Z}_{12-\mathrm{I}}$ orbifold with Wilson line. Nucl Phys B (2006) 770: 47-82. doi: 10.1016/j.nuclphysb.2007.02.008

35. Kim JE, Kim J-H, Kyae B. Superstring standard model from $Z_{12-I}$ orbifold compactification with and without exotics, and effective R-parity. JHEP (2007) 0706: 034. doi: 10.1088/1126-6708/2007/06/034

36. Huh J-H, Kim JE, Kyae B. SU(5) flip $x$ SU(5)' from $Z_{12-I}$. Phys Rev D (2009) 80: 115012. doi: 10.1103/PhysRevD.80.115012

37. Lebedev O, Nilles HP, Raby S, Ramos-Sanchez S, Ratz M, Vaudrevange PKS et al. A Mini-landscape of exact MSSM spectra in heterotic orbifolds. Phys Lett B (2007) 645: 88-94. doi: 10.1016/j.physletb.2006.12.012

38. Lebedev O, Nilles HP, Raby S, Ramos-Sanchez S, Ratz M, Vaudrevange PKS et al. The Heterotic Road to the MSSM with R parity. Phys Rev D (2008) 77: 046013. doi: 10.1103/PhysRevD.77.046013 
39. Nilles HP, Ramo-Sanchez S, Ratz M, Vaudrevange PKS. From strings to the MSSM. Euro Phys JC (2009) 59: 249-67. doi: 10.1140/epjc/s10052-0080740-1

40. Veneziano G, Yankielowitz S. An Effective Lagrangian for the Pure $\mathrm{N}=1$ Supersymmetric Yang-Mills Theory. Phys LettB (1982) 113: 231-36. doi: 10.1016/0370-2693(82)90828-0

41. Dixon L, Kaplunovsky V, Louis J, Peskin M. (1990). L. Dixon, talk presented at the A. P. S. D. P. F. Meeting at Houston; V. Kaplunovsky, talk presented at "Strings 90" workshop at College Station. Unpublished.

42. Casas JA, Lalak Z, Munoz C, Ross GG. Hierarchical Supersymmetry Breaking and Dynamical Determination of Compactification Parameters by Nonperturbative Effects. Nucl Phys B (1990) 347: 243-69. doi: 10.1016/05503213(90)90559-V

43. Kachru S, Schulz MB, Trivedi S. Moduli stabilization from fluxes in a simple IIB orientifold. JHEP (2003) 0310: 007. doi: 10.1088/1126-6708/2003/10/007

44. Goldberger WD, Wise MB. Modulus stabilization with bulk fields. Phys Rev Lett. (1999) 83: 4922-5. doi: 10.1103/PhysRevLett.83.4922

45. Nilles HP. Dynamically Broken Supergravity and the Hierarchy Problem. Phys Lett B (1982) 115: 193. doi: 10.1016/0370-2693(82)90642-6

46. Narain KS. New heterotic string theories in uncompactified dimensions $<10$. Phys Lett B (1986) 169: 41-6. doi: 10.1016/0370-2693(86)90682-9

47. Kakushadze Z, Tye $\mathrm{H}$. Three family $\mathrm{SO}(10)$ grand unification in string theory. Phys Rev Lett. (1996) 77: 2612-5. doi: 10.1103/PhysRevLett.77.2612
48. DeWolfe O, Hauer T, Iqbal A, Zwiebach B. Uncovering infinite symmetries on [p, q] 7-branes: Kac-Moody algebras and beyond. Adv Theor Math Phys. (1999) 3: 1835-91. arXiv: hep-th/9812209

49. Giveon A, Kutasov D. Brane dynamics and gauge theory. Rev Mod Phys. (1998) 71: 983-1084. doi: 10.1103/RevModPhys.71.983

Conflict of Interest Statement: The authors declare that the research was conducted in the absence of any commercial or financial relationships that could be construed as a potential conflict of interest.

Received: 15 October 2014; accepted: 11 December 2014; published online: 09 January 2015.

Citation: Choi K-Y, Kim JE and Kyae B (2015) Perspective on completing natural inflation. Front. Phys. 2:83. doi: 10.3389/fphy.2014.00083

This article was submitted to High-Energy and Astroparticle Physics, a section of the journal Frontiers in Physics.

Copyright (c) 2015 Choi, Kim and Kyae. This is an open-access article distributed under the terms of the Creative Commons Attribution License (CC BY). The use, distribution or reproduction in other forums is permitted, provided the original author(s) or licensor are credited and that the original publication in this journal is cited, in accordance with accepted academic practice. No use, distribution or reproduction is permitted which does not comply with these terms. 